\title{
The emergence of noncommutative target space in noncritical string theory
}

\author{
Jan Ambjørn ${ }^{a, c}$ and Romuald A. Janik ${ }^{b}$, \\ ${ }^{a}$ The Niels Bohr Institute, Copenhagen University \\ Blegdamsvej 17, DK-2100 Copenhagen Ø, Denmark. \\ email: ambjorn@nbi.dk \\ ${ }^{b}$ Institute of Physics, Jagellonian University, \\ Reymonta 4, PL 30-059 Krakow, Poland. \\ ${ }^{c}$ Institute for Theoretical Physics, Utrecht University, \\ Leuvenlaan 4, NL-3584 CE Utrecht, The Netherlands.
}

\begin{abstract}
We show how a noncommutative phase space appears in a natural way in noncritical string theory, the noncommutative deformation parameter being the string coupling.
\end{abstract}




\section{Introduction}

String theory should provide us with the dynamics and geometry of target space. In fact it should allow us to understand precisely what target space is and to what extent the classical concepts of space and time are valid. The discovery of branes and dualities in string theory has made this a vast topic.

Noncritical string theories with $c \leq 1$ defined via matrix models allow us to study various nonperturbative aspects of string theory. In particular they offer us a possibility to study the summation of string perturbation theory, which was why they attracted so much attention in the early 90ties. They play a role similar to the one that certain $2 \mathrm{~d}$ quantum field theories play in the study of $4 \mathrm{~d}$ quantum field theories: the possibility to solve them allows us to test nonperturbative concepts.

More recently the study of noncritical strings has again received much attention since they have a worldsheet formulation as conformal field theories coupled to Liouville field and an explicit matrix model formulation. Important progress has been made in both formulations in the understanding of $D$-branes and has resulted in a fruitful interplay between the two "dual" theories. In particular for $c<1$, where a target space formulation had been missing, the study of $D$-branes gave us an understanding of the target space via the study of the moduli space of the D-branes. The space of the so-called FZZT-branes is labeled by a single parameter $x$ and the disk amplitude $S(x)$ satisfies the following (semiclassical) equation [1, 2]

$$
T_{l}(W / C)=T_{k}(x), \quad W=\partial_{x} S(x)
$$

in the $(x, W)$ plane, where $C$ is a constant and $T_{p}$ are Chebyshev polynomials. Eq. (II) is valid for a minimal $(l, k)$ string theory in the so-called conformal closed string background. For future reference we note that in the special case of a minimal $(2,2 m-1)$ string theory, eq. (11) can be written as

$$
W^{2}=\left(P_{m}(x, u)\right)^{2}(x+u)
$$

where $P(x, u)$ is a polynomial of degree $m-1$ in $x$ and $u . u=\sqrt{\mu}$ where $\mu$ can be viewed as an effective cosmological constant and $x$ can be interpreted as a boundary cosmological constant. We also note that (2) is valid for any so-called closed string background, $u$ being a function $u(t)$ of a certain number of coupling constants $t_{i}, i=0, \ldots, m$, as will be explained later. 
From this point of view the semiclassical moduli space is a rather complicated Riemann surface (2) and in the ordinary complex plane $x$ the function $S(x)$ has cuts. This picture is true to all finite orders in string perturbation theory. In fact it is essential for the whole philosophy of string perturbation theory in the context of matrix models that the cut structure in the complex plane is unchanged. However, since it was first realized that the string perturbation theory for non-critical strings could be resummed it was also realized that the resummed solution to string theory (the non-perturbative partition function in the presence of the FZZT brane labeled by $x$ ) was an entire function in the complex $x$-plane [3]. The cut had disappeared and it was seemingly an artifact of the perturbative expansion. This point was reemphasized in a recent paper [4] in the context of eq. (11).

The purpose of this note is to explore what kind of geometrical structure should replace the Riemann surface (2) on the (nonperturbative) quantum level. In particular one has to give a meaning to the $W$ variable on the nonperturbative level since it does not make sense to consider disk amplitudes then. Promoting $W$ to an independent coordinate is also natural from the point of view of the link between $c<1$ strings and B-model topological string on the Calabi-Yau manifold

$$
z w=W^{2}-P^{2}(x)(x+u)
$$

where $W$ and $x$ (and $z, w)$ are (complex) coordinates. In this paper, however, we will not address this issue and stay purely within the context of $c<1$ strings. The 'quantum' $W$ and $x$ coordinates are in a completely natural way noncommutative while the vestige of the Riemann surface (2) on the quantum level is an additional projection operator.

In this note we make the above explicit - we point out that the disappearance of the Riemann surface (II) has a natural interpretation in terms of noncommutative geometry: For a non-zero string coupling one can define the concept of noncommutative phase space and in some sense, to be specified below, the Riemann surface (11) appears in the limit of vanishing string coupling which is also the limit where the noncommutative parameter goes to zero. The formulation also has the advantage that it brings the variables $x$ and $W$ in (11) on an equal footing as phase space variables, it describes in a natural way the "quantum mechanical" nature of target space and it connects to a similar (well known) description in the case of the $c=1$ string [5]. 
The integrable KdV structure of non-critical string theories is related to a formulation in terms of free fermions (as is the case for most integrable systems) and it is this fermionic structure which allow us in a natural way to introduce a noncommutative phase space.

In the next section we will present the noncommutative geometric structure, and we then study the explicit realization in $(2,2 m-1)$ non-critical string theory.

\section{The noncommutative geometry framework}

The $(2,2 m-1)$ models have a representation via one-matrix integrals. Before the continuum limit is obtained we can write the partition function as

$$
Z=\int d M \mathrm{e}^{-\frac{1}{g_{s}} \operatorname{Tr} V(M)}
$$

for a suitable potential $V(M)$. This matrix model can be solved using orthogonal polynomials $P_{n}(x)$ satisfying

$$
\int d x P_{n}(x) P_{m}(x) \mathrm{e}^{-\frac{1}{g_{s}} V(x)}=\delta_{n m}
$$

A free fermion description can be obtained by introducing fermions with wave functions

$$
\psi_{n}(x)=P_{n}(x) \mathrm{e}^{-\frac{1}{2 g_{s}} V(x)},
$$

i.e. we introduce a second quantized free fermion field

$$
\Psi(x)=\sum_{n=1}^{\infty} a_{n} \psi_{n}(x), \quad\left\{a_{m}, a_{n}^{\dagger}\right\}=\delta_{m, n} .
$$

The Fermi sea is defined by filling up the first $N$ levels, where $N$ is the size of the matrix, i.e.

$$
a_{n}|0\rangle=0 \quad \text { for } n \geq N, \quad a_{n}^{\dagger}|0\rangle=0 \quad \text { for } n<N
$$

Expectation values of traces of the matrix $M$ can now be calculated nicely in the free fermionic theory, i.e.

$$
\left\langle\operatorname{Tr} M^{k}\right\rangle=\left\langle N\left|\Psi^{\dagger} \hat{x}^{n} \Psi\right| N\right\rangle
$$


where $\hat{x}$ is the multiplication operator corresponding to $x$. The origin of the fermionic nature of $\Psi$ is the Vandermonde determinant resulting from the integration over the angular part of the matrix variables in the integral (4).

The wave function of the first unoccupied level is $\psi_{N}(x)$, which by the Heine formula for orthogonal polynomials can be written:

$$
\psi_{N}(x)=\langle\operatorname{det}(x-M)\rangle,
$$

where the expectation value is taken with respect to partition function $Z$ in (4). The continuum limit of the $(2,2 m-1)$ matrix model is obtained by taking a certain scaling limit when $N \rightarrow \infty$ and the variable $x$ is close to the cut of eigenvalues of the matrix model in the large $N$ limit. More explicitly one writes:

$$
x \rightarrow x_{c}+a x, \quad \frac{n}{N}=1-a^{m}\left(t_{0}-\tau\right), \quad N a^{m+\frac{1}{2}}=\frac{1}{g_{s}} .
$$

With this notation $\tau \rightarrow t_{0}$ means $n \rightarrow N$, and as will be described below, $t_{0}$ has the interpretation as the coupling constant related to a perturbation with the most dominant primary operator of the $(2,2 m-1)$ conformal field theory coupled to gravity, while $x$ has the interpretation of a boundary cosmological constant.

In this limit one obtains (see for instance [6] for a review)

$$
\psi\left(x_{c}+a x\right) \propto \psi(\tau, x),
$$

where the double-scaled function $\psi(\tau, x)$ satisfies

$$
Q \psi(\tau, x)=x \psi(\tau, x), \quad Q=g_{s}^{2} \frac{\mathrm{d}^{2}}{\mathrm{~d} \tau^{2}}-u\left(\tau, g_{s}\right) .
$$

Eq. (13) can be viewed as a Schrödinger-like equation with $-Q$ playing the role of the Hamiltonian, $u(\tau)$ the role of the potential and $-x$ the role of energy. It would allow for a standard WKB expansion in $g_{s}$ if it was not for the fact that the "potential" $u\left(\tau, g_{s}\right)$ was itself a function of $g_{s}$, determined from the so-called string equation

$$
[Q, P]=g_{s}, \quad P \psi(\tau, x)=g_{s} \frac{\mathrm{d}}{\mathrm{d} x} \psi(\tau, x)
$$

where $P$ is a polynomial in $d / d \tau$ and $u$. As is seen $Q$ and $P$, represent in a way the noncommutative phase space variables $q=x$ and $p=g_{s} d / d x$. $p$ is then 
to be identified with the $W$ coordinate on the (nonperturbative) quantum level. However, from (13) another (conceptually simpler) representation of a noncommutative phase space will emerge which we now turn to describe.

The important point for us at this stage is the notion of a Fermi sea, the ground state, in a theory of free fermions, above which we consider excitations. We thus have to project from wave functions all components below the Fermi level, which is explicitly done by the kernel

$$
K_{c<1}(x, y)=\sum_{n=N}^{\infty} \psi_{n}(x) \psi_{n}(y)
$$

It can be written as

$$
K_{c<1}(x, y)=\delta(x-y)-\sum_{n=0}^{N-1} \psi_{n}(x) \psi_{n}(y)=\delta(x-y)-K_{r m m}(x, y)
$$

where

$$
K_{r m m} \propto \frac{\psi_{N}(x) \psi_{N-1}(y)-\psi_{N-1}(x) \psi_{N}(y)}{x-y}
$$

is the standard random matrix kernel where an appropriate double scaling limit should be taken according to (11). Clearly a derivative with respect to $\tau$ is involved in the double scaling limit of (17) and using eq. (13) one obtains

$$
K_{c<1}\left(x, y ; t_{0}\right)=\int_{t_{0}}^{\infty} \mathrm{d} \tau \psi^{*}(\tau, x) \psi(\tau, y)
$$

The first unoccupied level is then exactly the Baker-Akhiezer function (or the 1-point function of the FZZT brane) as is clear from (101) and in the double scaling limit we can write $\psi\left(t_{0}, x\right)$.

The above construction fits nicely into the general framework of noncommutative geometry. Let the starting point be the noncommutative plane. It can be defined as the algebra $\mathcal{A}$ generated by $p$ and $q$ operators acting on $\mathcal{H}=L^{2}(\mathbb{R})$.

This Hilbert space in the context of $c<1$ strings is naturally identified with the space of wavefunctions (12) of the excitations of the random matrix model in the double scaling limit (we will discuss the Fermi sea in this context shortly).

In order to pass to the picture of functions of two variables (these will be the $p \equiv i W$ and $q \equiv x$ of the target space of the minimal string theory) with 
noncommutative multiplication one uses the Weyl map

$$
f(p, q) \longrightarrow \hat{f}=\int \frac{\mathrm{d} k_{1} \mathrm{~d} k_{2}}{(2 \pi)^{2}} \mathrm{e}^{i\left(k_{1} \hat{q}+k_{2} \hat{p}\right)} \tilde{f}\left(k_{1}, k_{2}\right),
$$

where $\tilde{f}$ is the Fourier transform of $f$. Eq. (19) maps functions to operators, and defines the noncommutative multiplication by

$$
\hat{f} \circ \hat{g} \rightarrow \longrightarrow f * g .
$$

The inverse map, sometimes called the Wigner map, is constructed by introducing via (19) the operator $\hat{\Delta}$ corresponding to the delta-function $\delta(q-$ $\left.q_{0}\right) \delta\left(p-p_{0}\right)$ :

$$
\hat{\Delta}\left(q_{0}, p_{0}\right)=\int \frac{\mathrm{d} k_{1} \mathrm{~d} k_{2}}{(2 \pi)^{2}} \mathrm{e}^{i\left(k_{1} \hat{q}+k_{2} \hat{p}\right)} \mathrm{e}^{-i\left(q_{0} k_{1}+p_{0} k_{2}\right)}
$$

and it is

$$
\hat{f} \longrightarrow f(q, p)=\operatorname{Tr}(\hat{f} \hat{\Delta}(q, p))
$$

The noncommutative product defined in this way by (20) is exactly equivalent to the standard Moyal product:

$$
f(q, p) * g(q, p)=f(q, p) \exp \left(i \frac{1}{2} \overleftarrow{\partial_{\mu}} \theta_{\mu \nu} \overrightarrow{\partial_{\nu}}\right) g(q, p)
$$

where $\mu, \nu$ refers to the $q, p$ coordinates and $\theta_{\mu \nu}=\theta \varepsilon_{\mu \nu}$ is the noncommutative parameter of the the theory.

At this stage we see that one ingredient of the $c<1$ string theory has not yet been incorporated so far. Since the elementary excitations are fermions and all the levels in the Fermi sea are already filled, the excitations $\psi(\tau, x)$ cannot probe the whole Hilbert space $\mathcal{H}=L^{2}(\mathbb{R})$ and one has to project out from $\mathcal{H}$ the states in the Fermi sea through (15). This projection leads to an additional geometrical structure in the noncommutative plane which is essentially the quantum nonperturbative analog of the classical Riemann surface (2) as we will show.

To this end let us note that associated with a given quantum wave function $\psi(x)$ we have the projection operator $|\psi\rangle\langle\psi|$ and the Wigner map applied to this operator gives the Wigner function $f_{\psi}(q, p)$ associated with the wave function $\psi(x)$ :

$$
f_{\psi}(q, p)=\int \frac{\mathrm{d} x}{2 \pi \hbar} \mathrm{e}^{i p x / \hbar} \psi^{*}(q-x / 2) \psi(q+x / 2) .
$$


where $q=\left(x_{1}+x_{2}\right) / 2$, the variables $x_{1}$ and $x_{1}$ being the coordinates which appear in the projector $\left\langle x_{2} \mid \psi\right\rangle\left\langle\psi \mid x_{1}\right\rangle$. Now we are ready to write the Wigner map associated with the projector

$$
\hat{\rho}=\sum_{n=N}^{\infty}\left|\psi_{n}\right\rangle\left\langle\psi_{n}\right|
$$

which leads to the associated Wigner function

$$
f_{\rho}(q, p)=\int \frac{\mathrm{d} x}{2 \pi \hbar} \mathrm{e}^{i p x / \hbar} \rho(q-x / 2, q+x / 2)
$$

where again $\rho\left(x_{1}, x_{2}\right)=\left\langle x_{1}|\hat{\rho}| x_{2}\right\rangle$ and $q=\left(x_{1}+x_{2}\right) / 2$.

The projection acts then on functions of two variables $q=x$ and $p=i W$ by the star multiplication:

$$
f(q, p) \longrightarrow f_{\rho}(q, p) * f(q, p)
$$

The geometrical structure which appears in the examples considered in this paper supplements the noncommutative plane by an appropriate (orthogonal) projection operator $\hat{K}$. One then truncates the space of functions to the image of $\hat{K}$ :

$$
\mathcal{H}_{K}=\left\{\hat{K} f: f \in L^{2}(\mathbb{R})\right\}
$$

and similarly the algebra of operators acting on $\mathcal{H}_{K}$ :

$$
\mathcal{A}_{K} \equiv\{\hat{K} \hat{O}: \hat{O} \in \mathcal{A}\}=\{\hat{K} \hat{O} \hat{K}: \hat{K} \in \mathcal{A}\}
$$

It is seen that this structure is precisely the one present also in $c=1$ non-critical string theory [5, 7, 8]. Let us now discuss in more detail the implementation of this abstract noncommutative geometrical structure in the $(2,2 m-1)$ minimal models.

Before we proceed with the general discussion let us illustrate the above notions in the simplest example of $(2,1)$ strings.

\section{$(2,1)$ minimal string theory}

In this case the Riemann surface is

$$
W^{2}-q-t_{0}=0
$$


Let us now proceed to find the projector which represents the geometry of the $(2,1)$ theory according to (26) . In this case the double scaling wavefunctions can be obtained exactly and we have

$$
\left(\hbar^{2} \frac{d^{2}}{d t_{0}^{2}}-t_{0}\right) \psi\left(t_{0}, x\right)=x \psi\left(t_{0}, x\right), \quad \psi\left(t_{0}, x\right)=\operatorname{Ai}\left(\frac{x+t_{0}}{\hbar^{2 / 3}}\right)
$$

while the Wigner transform is

$$
f_{\psi}(q, p)=\frac{1}{\pi \hbar^{2 / 3}} \operatorname{Ai}\left(\frac{p^{2}+\left(q+t_{0}\right)}{\hbar^{2 / 3}}\right)
$$

We see already here that the curve (30) appears as the argument of the Airy function. The Wigner transform of the projector is then obtained by integrating over $t_{0}$

$$
f_{\rho}(q, p)=\int_{t_{0}}^{\infty} \mathrm{d} t_{0}^{\prime} f_{\psi}\left(q, p ; t_{0}^{\prime}\right)
$$

Let us now examine the classical limit $\hbar \rightarrow 0$. Using the known asymptotics of integrals of Airy functions we have in the classically forbidden region

$$
f_{\rho}(q, p) \sim \frac{\hbar^{1 / 2}}{\left(p^{2}+q+t_{0}\right)^{3 / 4}} \exp \left(-\frac{2}{3 \hbar^{2 / 3}}\left(p^{2}+q+t_{0}\right)^{3 / 2}\right),
$$

while in the classically allowed region

$$
f_{\rho}(q, p) \sim 1-\frac{\hbar^{1 / 2}}{\sqrt{\pi}\left(-p^{2}-q-t_{0}\right)^{3 / 4}} \cos \left(\frac{2}{3 \hbar^{2 / 3}}\left(-p^{2}-q-t_{0}\right)^{3 / 2}+\frac{\pi}{4}\right) .
$$

Therefore the projector in this limit approaches the Heaviside step function

$$
f_{\rho}(q, p) \longrightarrow \theta\left(p^{2}+q+t_{0}\right)
$$

The Riemann surface curve appears then as the boundary of the classically allowed region which is natural since the FZZT 1-point function is the lowest excitation above the Fermi sea.

We will now proceed to explore these issues in the much more complicated case of $(2,2 m-1)$ minimal string theories.

First we outline how one obtains (11) in the semiclassical limit where $\hbar \equiv g_{s} \rightarrow 0$ from the Wigner-function corresponding to $\psi\left(t_{0}, x\right)$, viewed as a function of $x$, not $t_{0}$. 


\section{The semiclassical limit and beyond}

The semiclassical solution to $(2,2 m-1)$ non-critical strings is entirely determined by the disk-amplitude. It can be written as function of a number of coupling constants $t_{i}, i=0, m$, and the boundary cosmological constant $q$. For a given choice of $t_{i}$ the explicit formula for the semiclassical diskamplitude is 9 :

$$
S(q, t)=\frac{1}{2} \oint_{\infty} \frac{\mathrm{d} \Omega}{2 \pi i} V^{\prime}(t, \Omega) \log \left(\frac{1+\sqrt{\frac{q+u(t)}{\Omega+u(t)}}}{1-\sqrt{\frac{q+u(t)}{\Omega+u(t)}}}\right) .
$$

while the formula for the derivative of the disk amplitude w.r.t. the boundary cosmological constant is (using the string equation (41))

$$
W(q, t)=\frac{\partial S}{\partial q}=\frac{1}{2}\left(\oint_{\infty} \frac{\mathrm{d} \Omega}{2 \pi i} \frac{V^{\prime}(t, \Omega)}{(\Omega-q) \sqrt{\Omega+u(t)}}\right) \sqrt{q+u(t)}
$$

The (derivative of the) potential is defined by

$$
V^{\prime}(t, q)=\sum_{k=0}^{m} t_{k} q^{k-1 / 2}
$$

and the function $u(t)$ is determined by the string equation (14). It can be written as a differential equation in $u$ :

$$
\sum_{k=0}^{m} t_{k} R_{k}(u)=0
$$

where $R_{k}(u)$ denotes the so-called Gelfand-Dikii differential polynomials of $u$, considered as a function of $t_{0}{ }^{1}$. In the semiclassical limit (40) reduces to

$$
\sum_{k=0}^{m} c_{k} t_{k} u^{k}=0 \quad \text { or } \quad \oint_{\infty} \frac{\mathrm{d} \Omega}{2 \pi} \frac{V^{\prime}(\Omega, t)}{\sqrt{\Omega+u(t)}}=0,
$$

\footnotetext{
${ }^{1}$ The Gelfand-Dikii differential polynomials can be recursively defined by

$$
\frac{\mathrm{d}}{\mathrm{d} t_{0}} R_{k+1}(u)=\left(\frac{\hbar^{2}}{4} \frac{\mathrm{d}^{3}}{\mathrm{~d} t_{0}^{3}}-u \frac{\mathrm{d}}{\mathrm{d} t_{0}}-\frac{1}{2} \frac{\mathrm{d} u}{\mathrm{~d} t_{0}}\right) R_{k}(u), \quad R_{0}=1, \quad R_{1}=-\frac{u}{2} .
$$
}


where the $c_{k}$ 's are the power series coefficients of $(1+x)^{-1 / 2}$. In this semiclassical approximation $u(t)=\sqrt{\mu(t)}$ where $\mu$ is the effective cosmological constant of $2 \mathrm{~d}$ Euclidean quantum gravity coupled to a $(2,2 m-1)$ conformal field theory, expressed via the string equation as a function of the coupling constants $t_{i}$.

A choice of coupling constants $t_{i}$ is called a choice of closed string background and from (38) it is seen that the semiclassical disk amplitude can be written as

$$
W(x, t)=\frac{1}{2}\left(\sum_{l=0}^{m} t_{l} P_{l}(x, u)\right) \sqrt{x+u},
$$

where the polynomial multiplying $t_{l}$ is

$$
P_{l}(x, u)=\sum_{i=0}^{l-1} c_{k} u^{k} x^{l-1-k} .
$$

Similar remarks applies to $S(x, t)$ which has a similar representation as (42) with polynomials $\tilde{P}_{l}(x, u)$ one degree higher than $P_{l}(x, u)$.

Differentiating $S(x, t)$ w.r.t. $-t_{0}$ reproduces precisely the FZZT amplitude for an insertion of the primary operator of the most negative (gravitationally dressed) scaling dimension ${ }^{2}, \Delta_{r=1, s=m-1}=-(m-2) / 2$ :

$$
\frac{\mathrm{d} S(x, t)}{\mathrm{d} t_{0}}=\sqrt{x+u}
$$

We note that this formula implies that

$$
\begin{aligned}
S(x, t) & =\int_{t_{0}(x)}^{t_{0}} \mathrm{~d} t_{0}^{\prime} \sqrt{x+u\left(t_{0}^{\prime}, t_{1}, \ldots\right)} \\
W(x, t) & =\frac{1}{2} \int_{t_{0}(x)}^{t_{0}} \mathrm{~d} t_{0}^{\prime} \frac{1}{\sqrt{x+u\left(t_{0}^{\prime}, t_{1}, \ldots\right)}}
\end{aligned}
$$

where $u\left(t_{0}(x)\right)=-x$.

\footnotetext{
${ }^{2}$ Differentiating $S$ or $W$ after the other coupling constants $t_{i}$ produce expressions, also only depending on $u$ and $q$, with no explicit reference to $t_{i}$. However, in general the $t_{i}$ 's cannot for $0<i \leq m-2$ be identified directly with coupling constants related to the primary operators of scaling dimension $-(m-2+i) / 2$ due to operator mixing with the (gravitational) descendents of the operator corresponding to $t_{0}$.
} 
A full non-perturbative treatment involves the following steps: first solve the string equation (40) for $u$. Then solve the Schrödinger equation (13) for $\psi(\tau, x)$ and finally construct the projector (18). The Wigner transform either of $\psi(\tau, x)$ or the projector $K(x, y ; \tau)$ then provides us with variables $(q, p)$ which can be viewed as phase space variables which lives on a noncommutative phase space defined by the projector as described above. Let us now show that in a naive semiclassical $\left(\hbar \equiv g_{s} \rightarrow 0\right)$ limit we obtain formally the classical phase space $p=W(q)$, i.e. for the the choice of $t_{k}$ 's corresponding to a conformal background precisely (11).

Let us write the solution to (13) as:

$$
\psi\left(t_{0}, x\right)=\sqrt{R(x, u)} \mathrm{e}^{ \pm S\left(x, t_{0}\right)}, \quad \frac{\partial S}{\partial t_{0}}=\frac{1}{R} .
$$

The (13) can be written as

$$
\frac{\hbar^{2}}{4}\left(\left(\frac{\mathrm{d} R}{\mathrm{~d} t_{0}}\right)^{2}-2 R \frac{\mathrm{d}^{2} R}{\mathrm{~d} t_{0}^{2}}\right)+\left(x+u\left(t_{0}\right)\right) R^{2}=1 .
$$

Eqs. (47) and (48) is one version of the WKB-equations ${ }^{3}$, which are equivalent to the Schrödinger equation (13). In the WKB approximation where only the lowest order terms in $\hbar$ is kept in $R$ and $S$ we have from (48)

$$
R(x, u)=\frac{1}{\sqrt{x+u}}
$$

and we obtain $S$ from (47) by integrating from the classical turning point, i.e. the point $t_{0}(x)$ where $u\left(t_{0}(x), t_{1}, \ldots\right)=-x$,

$$
S(x, t)=\int_{t_{0}(x)}^{t_{0}} \mathrm{~d} t_{0}^{\prime} \sqrt{x+u\left(t_{0}^{\prime}, t_{1}, \ldots\right)} .
$$

\footnotetext{
${ }^{3}$ Note that if we differentiate (48) with respect to $t_{0}$ we obtain
}

$$
\left(\frac{\hbar^{2}}{4} \frac{\mathrm{d}^{3}}{\mathrm{~d} t_{0}^{3}}-(x+u) \frac{\mathrm{d}}{\mathrm{d} t_{0}}-\frac{1}{2} \frac{\mathrm{d} u}{\mathrm{~d} t_{0}}\right) R=0
$$

which shows that $R(x, u)$ is the generating functional for the Gelfand-Dikii polynomials:

$$
R(x, u)=\sum_{k=0}^{\infty} R_{k}(u) x^{-k-1 / 2} .
$$

It also follows from the definition that $R(x, u)$ is the diagonal element of the resolvent: $\left\langle t_{0}\left|(Q-x)^{-1}\right| t_{0}\right\rangle$. 
Comparing with (45) we see explicitly that the WKB approximation indeed reproduces the exponential of the semiclassical disk amplitude, i.e. the partition function of a single FZZT brane, as expected from the general discussion above.

\subsection{The naive limit}

We can now use this WKB approximation to calculate the Wigner transform:

$$
f_{\psi}(q, p)=\int \frac{\mathrm{d} x}{2 \pi \hbar} \mathrm{e}^{i p x / \hbar} \psi^{*}(q-x / 2) \psi(q+x / 2) .
$$

We emphasize that this Wigner transformed function is different from the ordinary Wigner function corresponding to $\psi_{x}\left(t_{0}\right)$ considered as an eigenfunction to the Schrödinger operator $Q$ with eigenvalue $x$. We perform the transformation in $x$, not in $t_{0}$, which is the variable appearing in $Q$.

Since $\hbar \rightarrow 0$ it is tempting (but not entirely correct as will be discussed shortly) to change integration variable $x=\hbar y$ and use the following approximations in the integrand:

$$
S(q+\hbar y / 2)=S(q)+\frac{\hbar y}{2} \frac{\partial S(q)}{\partial q}+O\left(\hbar^{2}\right)
$$

and $R(q+\hbar y / 2, u) R^{*}(q-\hbar y / 2)=|R(q, u)|^{2}+O\left(\hbar^{2}\right)$. If we first consider $q$ which is in the "classically allowed region" (where $S(q)$ is imaginary), and use eq. (52) in eq. (51) we obtain

$$
f_{\psi}(q, p)=|R(q, u(t))| \delta\left(p^{2}+\left(\frac{\partial S(q, t)}{\partial q}\right)^{2}\right)=\delta\left(t_{0}-t_{0}^{i}(q, p)\right),
$$

where $t_{0}^{i}(q, p)$ is defined as the value of $t_{0}$ where the argument of the $\delta$ function is zero for given, fixed values of $p$ and $q$. In the "classical forbidden region" (where $S(q)$ is real) we obtain in the same approximation zero because the contribution is exponentially suppressed by $\exp (-2 S(q) / \hbar)$ and $\hbar \rightarrow 0$.

From eq. (53) we finally obtain for the Wigner-transformed projector (see (26) and (18)

$$
f_{\rho}(q, p)=\theta\left(p^{2}+\left(\frac{\partial S(q, t)}{\partial q}\right)^{2}\right) .
$$

In the above semiclassical approximation we have thus seen that the Wigner transform gives us the classical phase-space (2), provided we make 
the identification $W \rightarrow i p$. This identification is indeed natural since the $W$ appearing in (2) should be viewed as a classical representative of the $P$ appearing in (14), i.e. a representation of the operator $d / d x$. Clearly, by construction, the $p$ appearing in the Wigner transform is a "real" $p$, related to $-i d / d x$.

\subsection{The WKB Wigner function}

The approximation (52) does not commute unfortunately with the integration in (51). Thus the results (53) and (54) are not correct, although they indeed lead to the correct semiclassical expectation values of nice observables like $\hat{x}^{n}$. A saddle-point calculation of (51) is however quite reliable except when the values of $q$ and $p$ are very close to the classical phase space orbit defined by (54). From (51) we obtain the saddle point equation for $x$ :

$$
W\left(q+x_{0} / 2\right)-W^{*}\left(q-x_{0} / 2\right)=2 i p
$$

In the classically allowed region (where $q+u(t)<0$ ) $W(q)$ is imaginary and eq. (55) allows for ordinary quantum mechanical systems a standard solution (see [10, 11, 12]) since the phase space curve is concave (see fig. 11), as emphasized in [10, 11, 12]. The saddle-point solution is

$$
f_{\psi}(q, p)=c^{2}\left(F(q, p) \exp \left(\frac{i}{\hbar} A(q, p)\right)+\text { c.c. }\right)
$$

where $c^{2}$ is a normalization factor irrelevant for the present discussion. The prefactor $F(q, p)$ is

$$
F(q, p)=\frac{\sqrt{R\left(q+\frac{x_{0}}{2}, t\right) R^{*}\left(q-\frac{x_{0}}{2}, t\right)}}{\sqrt{W_{x_{0}}^{\prime}\left(q+\frac{x_{0}}{2}\right)+W_{x_{0}}^{\prime}\left(q-\frac{x_{0}}{2}\right)}},
$$

where $x_{0}$ is the solution to (155) and $W_{x_{0}}^{\prime}$ denotes the derivative after $x_{0}$. Finally $A(q, p)$ is the value of the exponent in (53) for the solution $x_{0}$ of (55), i.e.

$$
A(q, p)=p x_{0}+S\left(q+\frac{x_{0}}{2}\right)+S\left(q-\frac{x_{0}}{2}\right) .
$$

For a concave phase space orbit $A$ and $(q, p)$ in the "interior", $A$ clearly has the interpretation of the area shown in fig. 1. If $W$ is imaginary then $-x_{0}$ is also a solution. Eq. (56) includes both saddle points via the complex conjugate term and $f_{\psi}(q, p)$ is real and oscillating. Note that these oscillations 


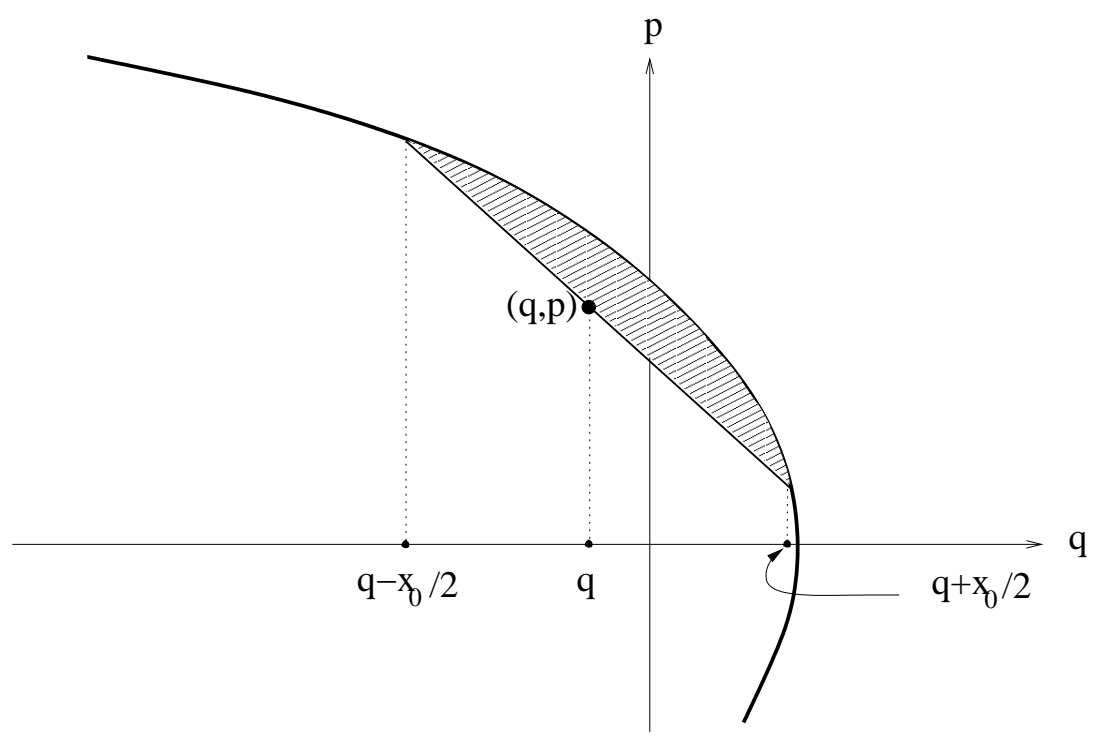

Figure 1: The graphical representation of the solution to eq. (155), the shaded area $A(q, p)$ being defined in eq. (58).

do not disappear in the limit $\hbar \rightarrow 0$. For an ordinary quantum mechanical system with $(q, p)$ outside the classically allowed region in phase space corresponding to a certain energy eq. (55) will have a complex solution $x_{0}$ and $f_{\psi}(q, p)$ will fall off exponentially.

If eq. (55.) holds for values of $(q, p)$ where $x_{0} \rightarrow 0$, i.e. when we approach the "classical phase space" defined by (54), we see that (56) diverges due to the divergence of the prefactor $F(q, p)$ for $x_{0} \rightarrow 0$. This is not related to the question of the reliability of the WKB approximation of $\psi\left(t_{0}, x\right)$ at the classical turning point, but just to a failure of the quadratic saddle point calculation of the Wigner integral and one can improve the calculation by the method of uniform approximation, which involves in a natural way the Airy functions. This was done in [1] where a closed expression was given, which is not only valid near the classical turning points but in the whole phase space, and which reduces to (56) away from the classical orbit:

$$
f_{\psi}^{w k b}(q, p)=\frac{c^{2}}{\hbar^{2 / 3}} F(q, p) A^{\frac{1}{6}}(q, p) \operatorname{Ai}\left(-\left[\frac{3 A(q, p)}{2 \hbar}\right]^{2 / 3}\right)
$$

where $c$ is a normalization constant independent of $\hbar$, irrelevant for the present discussion. The Wigner WKB-function is a good approximation to 
the exact result for small $\hbar$, in the sense that it incorporates already the improvements to the simple WKB approximation present in the so-called uniform semiclassical approximation even if the starting point was the simple WKB-approximation for $\psi$ [1]. In particular, it is finite at the classical orbit except in exceptional cases discussed in [11].

Again, the non-disappearance of $f_{\psi}(q, p)$ in the classically allowed region but away from the classical orbit is quite manifest in the expression (59). The following features are noteworthy: (1) the peak of $f_{\psi}(q, p)$ grows as $\hbar^{-2 / 3}$ as $\hbar \rightarrow 0$. Thus $f_{\psi}(q, p)$ a priori had a chance to reproduce the $\delta$-function at the classical orbit (53). However the Airy function oscillates in this region and these oscillations also diverge in the limit $\hbar \rightarrow 0$ although slightly weaker. Folded with sufficiently smooth functions these oscillations average out, and for "observables" $O(q, p)$ corresponding to such functions the $\hbar \rightarrow 0$ limit of $f_{\psi}(q, p)$ will indeed be given by (53) (see [10] for discussion and examples). (2) Since the peak of the Airy function is not at zero it follows from (59) that the "fuzzy classical orbit" is actually displaced a distance proportional to $\hbar^{2 / 3}$ and the spread of this fuzzy orbit is also of order $\hbar^{2 / 3}$.

\subsection{Beyond the semiclassical limit (almost)}

The simplest example is provided by the $(2,1)$ minimal string model $(m=1)$. In this case we have simply $u(t)=t_{0}$. Thus we can explicitly solve the system both in the semiclassical limit and completely non-perturbatively in $\hbar$.

Let us first state form of the WKB-approximation above. Let us denote $-t_{0}$ by $E$, the "classical energy". Thus we have on a classical orbit

$$
p_{c l}^{2}+q_{c l}=E, \quad \frac{3}{2} A(q, p)=\left(E-\left(p^{2}+q\right)\right)^{3 / 2},
$$

while $F(q, p) \propto A^{-1 / 6}(q, p)$, and we obtain from (59)

$$
f_{\psi}^{w k b}(q, p) \propto \frac{1}{\hbar^{2 / 3}} \operatorname{Ai}\left(\frac{p^{2}+q+t_{0}}{\hbar^{2 / 3}}\right) .
$$

The exact solution was already cited earlier (31) and its Wigner transform is

$$
f_{\psi}(q, p)=\frac{1}{\pi \hbar^{2 / 3}} \operatorname{Ai}\left(\frac{p^{2}+\left(q+t_{0}\right)}{\hbar^{2 / 3}}\right)
$$

Maybe not surprisingly the Airy-function uniformized WKB approximation is exact for the linear potential! However, it suggests that many of the 
lessons one can draw from (159) might well be valid beyond the semiclassical approximation. At least here we have seen that in the context of the simplest non-critical string theory it includes a summation over all genera.

Given the explicit expression (32) we can calculate the Wigner transform $f_{\rho}(q, p)$ of the projector $K$. The projector $f_{\rho}(q, p)$ is better behaved than $f_{\psi}(q, p)$ w.r.t. the classical limit. As shown in (34)-(35) the projector $f_{\rho}(q, p)$ indeed approaches the naive limit (53) for $\hbar \rightarrow 0$.

This argument can clearly be extended to the general WKB Wigner transform given by (59). Integrating with respect to $t_{0}$ will produce additional factors of $\hbar$ which will suppress the oscillations in the "classically allowed region" in the limit $\hbar \rightarrow 0$.

\section{Discussion}

Non-critical string theory has always been a useful laboratory for the study new ideas in string theory. Most recently it has received renewed attention after it was realized that the concepts of branes and open-closed string duality could be analyzed using matrix model technology. In this connection a target space interpretation of the the $c<1$ non-critical strings was suggested, target space being identified with a Riemann surface which was explicitly constructed in the conformal background for a $(k, l)$ non-critical string in the semiclassical approximation.

One of the virtues of non-critical string theory is that it, at least to some extent, offers us a non-perturbative definition of string theory. In particular for the $(2,2 m-1)$ non-critical string theories, where $m$ is odd, this is relatively concrete: one can define a theory which should represent the nonperturbative sum over all genera in the sense that one can actually solve the string equation and find a "physically acceptable" string susceptibility function $u(t)$ well defined on the whole real $t_{0}$-axis [13. One problem arises in this connection: the elaborate structure of target space seemingly present semiclassically disappears completely and target space reduces to the complex plane. It seems a quite unsatisfactory situation if we extrapolate back to critical string theory. It would imply that we know nothing about target space unless we are able to perform the sum over all genera in critical string theory. It prompted us to look for another target space interpretation of the non-critical strings. We were here inspired by the fact that in $c=1$ noncritical string theory (where the notion of target space is more clear than for 
non-critical string theories with $c<1$ ) the concept of a non-commutative phase space appears naturally [5].

In this note we have shown that noncommutative phase space indeed appears in a natural way in non-critical string theory. The Wigner function associated with the projector which defines the non-commutative phase space reduces in the semiclassical limit precisely to (54). It also makes it possible to avoid taking about Riemann surfaces altogether, if wanted. Target space becomes associated with a (real) coordinate $q$ to which we can associate a noncommutative "momentum" $p$. Since we consider $c<1$ we have no time evolution in this target space but orbits of constant "energy" makes sense and some aspects of the noncommutative nature of the phase space survive even semiclassically since the WKB approximation precisely defines the semiclassical FZZT brane.

Let us perhaps contrast the appearance of noncommutative geometry in these theories with the earlier studies of noncommutativity in the context of backgrounds with nonzero $B_{N S-N S}$ and the resulting open string theories [14. There the noncommutativity was a property of a particular background and could be turned off at will, while here it is intrinsically linked to the string coupling constant.

Let us note that the same noncommutative geometrical structure appears naturally also in other contexts in string theory. $c=1$ strings also have a free fermion formulation with a filled Fermi sea whose classical boundary is the curve $p^{2}-q^{2}-\mu=0$. In an analogous fashion the quantum description will again be the noncommutative plane with a projection operator which represents a fuzzy noncommutative version of the Fermi sea.

Since the $c<1$ and $c=1$ string theories are closely associated with B-model topological strings on Calabi-Yau's of the form $z w-H(p, q)=0$, the above results also suggest that similar noncommutative structures should also appear in that context.

It would be also interesting to explore the issues of noncommutativity in the context of the $\frac{1}{2}$-BPS sector in the AdS/CFT correspondence which on the classical level is mapped to a free fermion description [15] and the corresponding classical geometry is encoded by the geometry of the Fermi sea [16].

Finally on a much more speculative level let us mention A-model topological strings formulated through the sum over 3D partitions [17. The blown-up Calabi-Yau's (associated to toric diagrams $\sim 3 \mathrm{D}$ partitions) are represented by a lattice of points in an octant. The missing points are very naturally 
identified with harmonic oscillator eigenfunctions which are projected out ${ }^{4}$. The melting crystal partition function would then have an interpretation as a summation over (admissible) quantum geometries represented by projectors.

It would be fascinating to understand more deeply the interrelations between all these pictures.

\section{Acknowledgment}

RJ was supported in part by KBN grants 2P03B08225 (2003-2006) and 1P03B02427 (2004-2007).

Note added: As this paper was being finished the work [18 appeared which also addresses the quantum phase space of minimal strings.

\section{References}

[1] N. Seiberg and D. Shih, Branes, rings and matrix models in minimal (super)string theory, JHEP 0402 (2004) 021 arXiv:hep-th/0312170.

[2] D. Kutasov, K. Okuyama, J. w. Park, N. Seiberg and D. Shih, Annulus amplitudes and ZZ branes in minimal string theory, JHEP 0408 (2004) 026 arXiv:hep-th/0406030.

[3] F. David, Loop Equations And Nonperturbative Effects In TwoDimensional Quantum Gravity, Mod. Phys. Lett. A 5 (1990) 1019;

F. David, Phases Of The Large $N$ Matrix Model And Nonperturbative Effects In 2-D Gravity, Nucl. Phys. B 348 (1991) 507.

[4] J. Maldacena, G. W. Moore, N. Seiberg and D. Shih, Exact vs. semiclassical target space of the minimal string, JHEP 0410 (2004) 020 arXiv:hep-th/0408039.

[5] A. Dhar, G. Mandal and S. R. Wadia, "Nonrelativistic fermions, coadjoint orbits of W(infinity) and string field theory at $c=1$," Mod. Phys. Lett. A 7 (1992) 3129 arXiv:hep-th/9207011;

G. Mandal and S. R. Wadia, Rolling tachyon solution of two-dimensional string theory, JHEP 0405 (2004) 038 arXiv:hep-th/0312192.

\footnotetext{
${ }^{4}$ E.g. such an interpretation for the toric diagram of $\mathbb{P}^{1}$ yields the fuzzy sphere.
} 
[6] P. H. Ginsparg and G. W. Moore, Lectures on 2-D gravity and 2-D string theory, arXiv:hep-th/9304011.

[7] A. Sen, Open-closed duality: Lessons from matrix model, Mod. Phys. Lett. A 19 (2004) 841 arXiv:hep-th/0308068.

[8] J. Ambjorn and R. A. Janik, The decay of quantum D-branes Phys. Lett. B 584 (2004) 155 arXiv:hep-th/0312163.

[9] J. Ambjorn, B. Durhuus and T. Jonsson, Quantum geometry. A statistical field theory approach, Cambridge Monogr. Math. Phys. 1 (1997) 1.

[10] E. J. Heller, Phase space interpretation of semiclassical theory, Journ. Chem. Phys. 67 (1977) 3339.

[11] M. V. Berry, semiclassical mechanics in phase space: a study of Wigner's function, Phil. Trans. R. Soc. London, 287 (1977) 237.

[12] S. Habib, The Classical Limit In Quantum Cosmology. 1 Quantum Mechanics And The Wigner Function, Phys. Rev. D 42, (1990) 2566;

S. Habib and R. Laflamme, Wigner Function And Decoherence In Quantum Cosmology, Phys. Rev. D 42, 4056 (1990).

[13] M. R. Douglas, N. Seiberg and S. H. Shenker, Flow And Instability In Quantum Gravity, Phys. Lett. B 244, 381 (1990).

[14] N. Seiberg and E. Witten, "String theory and noncommutative geometry,” JHEP 9909 (1999) 032 arXiv:hep-th/9908142.

[15] D. Berenstein, "A toy model for the AdS/CFT correspondence," JHEP 0407, 018 (2004) arXiv:hep-th/0403110.

[16] H. Lin, O. Lunin and J. Maldacena, "Bubbling AdS space and 1/2 BPS geometries," JHEP 0410 (2004) 025 arXiv:hep-th/0409174.

[17] A. Okounkov, N. Reshetikhin and C. Vafa, "Quantum Calabi-Yau and classical crystals," arXiv:hep-th/0309208;

A. Iqbal, N. Nekrasov, A. Okounkov and C. Vafa, "Quantum foam and topological strings," arXiv:hep-th/0312022. 
[18] C. G. Gomez, S. Montanez and P. Resco, Semi-classical mechanics in phase space: the quantum target of minimal strings, arxiv:hep-th/05066159]. 